\title{
Point-of-care ultrasonography: a practical step in the path to precision in critical care
}

\author{
Gentle Sunder Shrestha
}

Management of critically ill patients is challenged by the complexity of underlying pathophysiology, ambiguity of illness syndromes, and rapidly changing physiological status. These unique characteristics of critical illness also justify the need for precision medicine, rather than applying a "one-size-fits all" approach in the ICU [1]. Many large clinical trials, even though supported by strong pre-clinical and pathopysiological bases, have failed to show clinical benefit, probably due to enrollment of heterogeneous patient populations and failure to identify endotypes [2].

Although it is an intriguing concept and there is a need for it, multiple challenges need to be overcome before the application of precision medicine in the ICU in clinical practice [1]. Designing future trials to test individualized therapy, developing and validating biomarkers, and analysis and interpretation of big data are time-consuming and expensive and may not be practical.

For a test or investigation to be practically applicable in the ICU, it should be applicable at the point-of-care, inexpensive, simple, and be feasible and repeatable at the bedside with a rapid turnover time. Critical care echocardiography (CCE) has been shown to be a valuable non-invasive bedside tool for the management of shock. It helps in the rapid evaluation of physiological derangement in patients with shock, to guide therapy, and to evaluate the response to treatment in real time [3]. When combined with critical care ultrasonography (CCUS), CCE is beneficial for rapid evaluation of acute respiratory failure, for adjusting ventilator setting and PEEP titration in ARDS, for identification of patients at risk for weaning failure, for diagnosis of pleural and lung pathologies, and thus for individualize patient management at the bedside [4].

International statements on training standards for CCUS recommend basic CCE and general CCUS to be essential requirements of ICU training programs. CCUS

Correspondence: gentlesunder@hotmail.com

Department of Anaesthesiology, Critical Care Unit, Tribhuvan University

Teaching Hospital, Maharajgunj, Kathmandu, Nepal is suggested to be the primary point-of-care imaging modality in the ICU. With the availability of cheaper, compact, and portable ultrasound units, its potential benefit and applicability seem greater and more pragmatic considering the cost and lack of imaging modalities like CT or MRI in resource-poor settings. The approach of whole-body point-of-care ultrasonography (POCUS) can be a practical and viable step in the path to precision in the ICU. The findings of bedside wholebody POCUS in a non-organ-specific manner are integrated with the findings of overall clinical assessment of the patient to individualize patient management [5].

\section{Abbreviations \\ ARDS: Acute respiratory distress syndrome; CCE: Critical care echocardiography; CCUS: Critical care ultrasonography; CT: Computerized tomography; ICU: Intensive care unit; MRI: Magnetic resonance imaging;} PEEP: Positive end expiratory pressure; POCUS: Point-of-care ultrasonography

\section{Acknowledgements \\ None.}

Funding

None.

Availability of data and materials

Not applicable.

\section{Authors' contributions}

GSS contributed to concept, designing, literature search, manuscript writing, editing, and review.

Ethics approval and consent to participate

Not applicable.

Consent for publication

Not applicable.

Competing interests

The author declares that he/she has no competing interests.

\section{Publisher's Note}

Springer Nature remains neutral with regard to jurisdictional claims in published maps and institutional affiliations.

Published online: 16 August 2017

\section{References}

1. Maslove DM, Lamontagne F, Marshall JC, Heyland DK. A path to precision in the ICU. Crit Care. 2017;21:79. 
2. Shrestha GS. Intensive blood-pressure lowering in cerebral hemorrhage. New Engl J Med. 2016;375:e48.

3. McLean AS. Echocardiography in shock management. Crit Care. 2016;20:275.

4. Vignon P, Repesse X, Vieillard-Baron A, Maury E. Critical care ultrasonography in acute respiratory failure. Crit Care. 2016;20:228.

5. Mayo P, Arntfield R, Balik M, et al. The ICM research agenda on critical care ultrasonography. Intensive Care Med. 2017. doi:10.1007/s00134-017-4734-z. 